\begin{tabular}{|c|l|}
\hline Title & Enhanced Photon Extraction from a Quantum Dot Induced by a Silver Microcolumnar Photon Reflector \\
\hline Author(s) & $\begin{array}{l}\text { Kumano, Hidekazu; Nakaj ima, Hideaki; lijima, Hitoshi; Odashima, Satoru; Matsuo, Y asuhiro; Ijiro, Kuniharu; } \\
\text { Suemune, Ikuo }\end{array}$ \\
\hline Citation & $\begin{array}{l}\text { A pplied Physics Express, 6(6), 062801-1-062801-4 } \\
\text { https://doi.org/10.7567/APEX .6.062801 }\end{array}$ \\
\hline Issue Date & 2013-06 \\
\hline Doc URL & http://hdl.handle.net/2115/59641 \\
\hline Rights & ○ 2013 The Japan Society of A pplied Physics \\
\hline Type & article (author version) \\
\hline File Information & APL6(2013).pdf \\
\hline
\end{tabular}

Instructions for use 


\title{
Enhanced Photon Extraction from a Quantum Dot Induced by a Silver Microcolumnar Photon Reflector
}

\author{
Hidekazu Kumano*1, Hideaki Nakajima ${ }^{1,2}$, Hitoshi lijima ${ }^{1}$, Satoru Odashima ${ }^{1}$, Yasuhiro \\ Matsuo $^{1}$, Kuniharu ljiro ${ }^{1}$, and Ikuo Suemune ${ }^{1}$ \\ ${ }^{1}$ Research Institute for Electronic Science, Hokkaido University, Sapporo 001-0021, Japan \\ ${ }^{2}$ Research Fellow of the Japan Society for the Promotion of Science, Tokyo 102-8472, Japan
}

\begin{abstract}
We have fabricated silver microcolumnar photon reflectors to enhance the photon extraction efficiency from a quantum dot. The mechanically robust planar structure is favorable to couple to optical fibers for stable and efficient single-photon sources. A high photon extraction efficiency of up to $18 \%$ is achieved. Furthermore, strong suppression of multiphoton generation is confirmed. The proposed structure is quite promising toward the implementation of practical quantum key distribution systems with dot-fiber-coupled photon sources.
\end{abstract}

Quantum key distribution (QKD) is now regarded as a crucial public infrastructure for absolutely secure communications, and long-distance fiber-based QKD experiments have been intensively carried out. ${ }^{1,2)}$ Photon sources are key devices in the QKD, and from the viewpoint of transmission length, nonclassical single photon sources (SPSs) based on semiconductor quantum dots (QDs) have an inherent advantage over the weak coherent pulse (WCP). ${ }^{3,4)}$ In order to guarantee the absolute secure communication with the SPSs, suppression of multiphoton emission is essential. Furthermore, a high photon extraction efficiency is important for practical use. In this regard, realization of SPSs with exceeding $10 \%$ efficiency can be a yardstick to have advantage over the WCP, which provides optical pulses containing a single photon with a probability of $9 \%$ under the widely used average photon number per pulse of 0.1 .

Up to now, a variety of SPS structures have been proposed for enhancing the photon extraction efficiency from QDs, such as horn structure, ${ }^{5)}$ photonic crystal, ${ }^{6,7)}$ microcavity, ${ }^{8,9)}$ tapered waveguide, ${ }^{10-13)}$ micro-pyramid, ${ }^{14)}$ and tamm plasmon modes. ${ }^{15)}$ As practical sources utilized in the fiber-based QKD, generated photons should be coupled into a single-mode fiber, and it is expected that firm contact between the source and the

*E-mail address: kumano@es.hokudai.ac.jp 
fiber is beneficial with respect to long-term stability. Some prototypes in this direction are reported in refs. 16 and 17. However most of the structures so far reported have a high structural aspect ratio; thus, these are not necessarily stable and not very appropriate for the optical fiber contact. In order to introduce the structural robustness for the fiber contact, we have proposed a planar SPS structure with a metal microcolumnar photon reflector. By fabricating the metal reflector, a QD ensemble embedded in semiconductor pillars showed 40-fold emission enhancement. ${ }^{18)}$

Fabrication of the proposed planar SPS structure included a cleavage process of the two-dimensional pillar arrays at the base of the pillars. For this cleavage purpose, the weak contact between a thin $\mathrm{Au}$ layer and a $\mathrm{SiO}_{2}$ surface was employed, which was inserted at the metal/semiconductor interface. However the "weak" contact was not satisfactory and the fabrication yield of the proposed SPS structures remained low. Another limitation was the employment of hard metals such as $\mathrm{Ti}$ and $\mathrm{Nb}$ from the requisite for the fabrication process. The cleavage process induces shear stress to the pillars, and hard metals were necessary for relatively short ( $\sim 300 \mathrm{~nm}$ high $)$ pillars. However, hard metals are known to have low optical reflectivity, and the photon extraction efficiency was limited to $8 \%{ }^{19)}$

In this paper, we newly introduce a removal layer for separating the metal reflector layer from the substrate, and a much improved fabrication yield as high as $70 \%$ is achieved. Furthermore a redesigned pillar structure with higher aspect ratio enables us to adopt highly reflective silver (Ag) as the microcolumnar photon reflector, leading to the high photon extraction efficiency up to $18 \%$.

InAs QDs were grown on a GaAs (001) substrate by metalorganic molecular beam epitaxy (MOMBE). The density of the InAs QDs is estimated as $\sim 3.5 \times 10^{9} \mathrm{~cm}^{-2}$, and the sample surface is terminated by a 50-nm-thick GaAs capping layer. After the growth, pillar structures were fabricated by electron-beam (EB) lithography and inductively coupled plasma reactive ion etching (ICP-RIE). Instead of the previously employed pillars of $300 \mathrm{~nm}$ height, ${ }^{19)}$ we prepared pillar structures of $750 \mathrm{~nm}$ height for the following cleavage process. Then, a $5 \mathrm{~nm} \mathrm{SiO}_{2}$ layer was deposited by plasma-enhanced chemical-vapor deposition (PECVD) and a 5-nm-thick silane coupling agent (Siliconize L-25) was coated as a removal layer [Fig. 1(a)]. Next, 3- $\mu$ m-thick Ag was deposited to cover whole pillar structures [Fig. 1(b)], and the Ag surface is glued to a supporting glass substrate with UV-cured resin [Fig. 1(c)]. The whole structure was turned upside down, and the pillars containing QDs in the Ag reflector layer were cleaved from the 
(a)

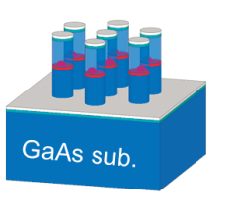

(c)

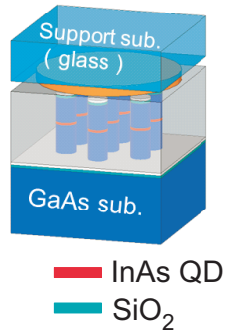

(e)

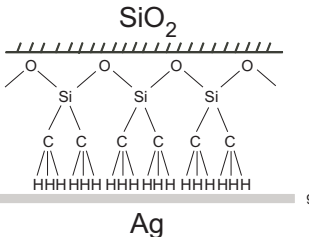

(b)

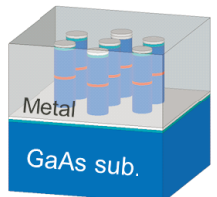

(d)

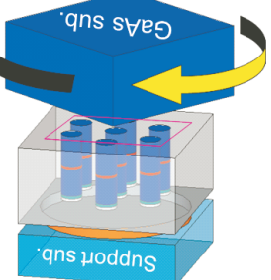

Silane coupling agent

UV-cured resin

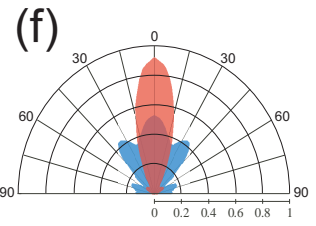

Fig. 1. (a)-(d) Fabrication process of planar single-photon sources with highly reflective silver microcolumnar photon reflectors. (e) Schematic of the $\mathrm{SiO}_{2} /$ silane coupling agent/Ag interfaces, which realizes high fabrication yield in removing the GaAs substrate in (d). (f) FDTD simulation of angle-resolved field intensity in airside using a micro-pillar structure with diameters of $400 \mathrm{~nm}$ (red area) and $500 \mathrm{~nm}$ (blue area). Center of the micropillar on the top plane is located at the origin of the polar chart. Coupling angle for an objective lens $(\mathrm{NA}=0.4)$ corresponds to $24^{\circ}$.

GaAs substrate by applying rotational shear stress [Fig. 1(d)]. Figure 2 shows the SEM images of a two-dimensional array of (a) prepared GaAs pillars before Ag deposition and (b) completed SPSs with Ag photon reflector. The baseplane of each pillar can be observed on the Ag surface in Fig. 2(b). The silane coupling agent is adhesive to the $\mathrm{SiO}_{2}$ layer due to the strong polar bond of oxygen, while weakly bonded to the Ag surface with hydrophobic hydrocarbon terminations [Fig. 1(e)]. Hence, the silane coupling agent assists the smooth separation of the Ag surface from the GaAs substrate and also sharp cleavage of the pillar structures at the base. Along with the redesigned pillar structure with higher aspect ratio, the fabrication yield of the photon sources has much improved to as high as $70 \%$. The $\mathrm{SiO}_{2}$ layer in between $\mathrm{Ag}$ and GaAs layers is also helpful to circumvent the formation of process-induced nonradiative recombination centers at the interface.

The photon extraction efficiency $\eta_{\text {ext }}$ from the prepared photon sources was examined at $20 \mathrm{~K}$. As an optical excitation source, a mode-locked Ti:sapphire laser (76.2 


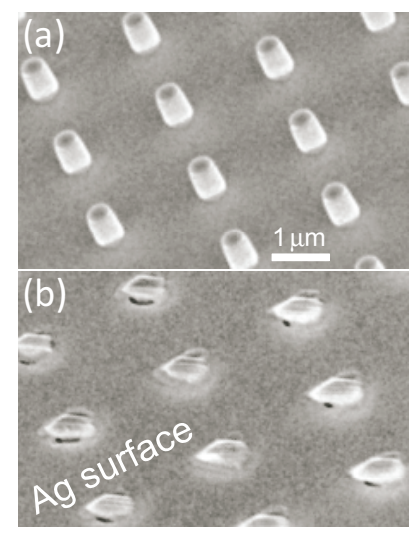

Fig. 2. SEM images of a two-dimensional array of (a) GaAs pillars formed by ICP-RIE and (b) SPSs with Ag photon reflector after removing GaAs substrate [cf. Fig. 1(d)].

$\mathrm{MHz}$ ) was used. The pumping energy was $1.5498 \mathrm{eV}$, which corresponds to the GaAs barrier excitation. The laser beam was focused on a single photon-source structure with an objective lens $(\mathrm{NA}=0.4)$. Negatively charged exciton $\left(X^{-}\right)$was predominantly formed [Fig. 3(a)] due to the preferential electron injection into the QD after electronhole pair generation by the GaAs barrier excitation, ${ }^{20)}$ and this single sharp line (centered around $1.2958 \mathrm{eV}, \sim 55 \mu \mathrm{eV}$ in FWHM) was filtered with a combination of twofold narrow band-pass, short-pass, and twofold long-pass filters (BPFs, SPF, and LPFs, respectively). Due to the twofold BPFs, the transmitted bandwidth is $\sim 0.3 \mathrm{~nm}$ in FWHM (each filter has $\sim 0.5 \mathrm{~nm}$ bandwidth), and the SPF and LPFs were used to suppress uncorrelated emission that originated from neighboring QDs as well as stray light from the laser source. The photons that passed through all the optics generated by the $X^{-}$ transition were finally detected by single-photon counting modules (SPCMs), and the single-photon emission rate and correlation function based on a Hanbury Brown and Twiss (HBT) interferometer setup ${ }^{21)}$ were evaluated.

Triangles in Fig. 3(b) show raw count rates measured by one of the SPCMs. Net single-photon rate is obtained by eliminating the multiphoton contribution by a factor of $\sqrt{1-g^{(2)}(0)},{ }^{9)}$ and displayed by squares. The $g^{(2)}(0)$ values are obtained from the photon correlation function analyzed with a multichannel scalar (MCS). The net singlephoton rate agrees well with $I_{0} \cdot \eta_{\{X \geq 1\}}$, which is given by the solid curve in Fig. 3(b). Here $I_{0}$ indicates an upper limit of the single-photon rate of $\sim 69.8 \mathrm{kHz}$ at $5.2 \mu \mathrm{W}$, obtained from the corresponding raw count rate $(84.0 \mathrm{kHz})$ and $g^{(2)}(0)=0.31$ as derived from Fig. 3(c). The $\eta_{\{X \geq 1\}}$ is a probability of more than one exciton being captured in the QD by each excitation event, and nonzero $\eta_{\{X \geq 1\}}$ gives rise to single photon 


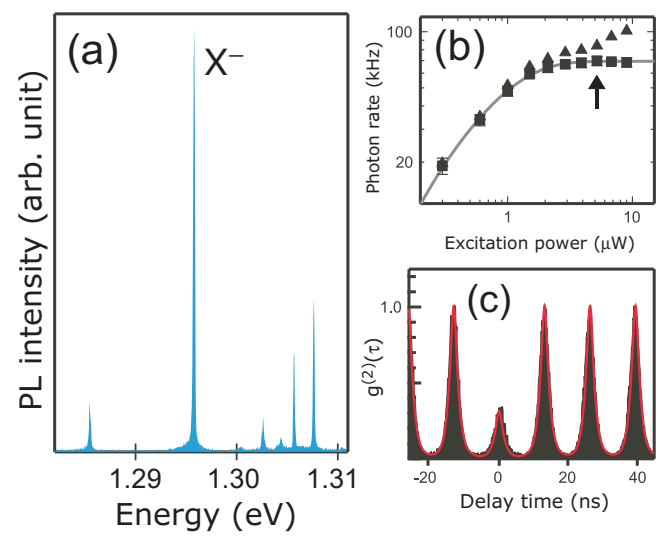

Fig. 3. (a) $\mu$-PL spectrum from a $\mathrm{QD}$ with $\mathrm{Ag}$ photon reflector under excitation power of $0.3 \mu \mathrm{W}$. (b) Excitation power dependence of raw detection rate (triangles) by one of the SPCMs and the net single-photon rate (squares) of the $X^{-}$line. The theoretical net single-photon rate expected based on Poissonian exciton population in a QD is given by a solid line. The arrow indicates excitation power at which the autocorrelation function (shown below) was measured and the $\eta_{\text {ext }}$ was evaluated. (c) Second-order autocorrelation function $g^{(2)}(\tau)$ measured at a saturated single-photon emission rate $(5.2 \mu \mathrm{W})$. Fitted results are also shown by a red curve, giving rise to $g^{(2)}(0)=0.31$.

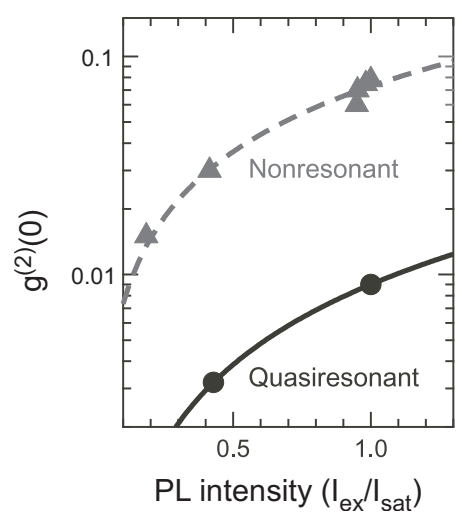

Fig. 4. $g^{(2)}(0)$ values as a function of excitation power with nonresonant (triangles) and quasiresonant (2LO) excitation (circles). Drastic suppression ( one order of magnitude) of multiphoton emission is observed for the quasiresonant excitation.

emission per pulse via the $X^{-}$transition with the spectral filtering. Assuming that the initial exciton population generated by each excitation cycle obeys the Poissonian distribution with the average exciton number of $\bar{N}$, we have $\eta_{\{X \geq 1\}}=1-\exp (-\bar{N}) .{ }^{22)}$ The net single photon rate corresponds to $f \eta_{\{X \geq 1\}} \eta_{\text {ext }} \eta_{\text {trans }} \eta_{\mathrm{SPCM}} \sec ^{-1}$, where $f, \eta_{\text {trans }}$, and $\eta_{\mathrm{SPCM}}$ denote the laser pulse repetition rate, transmission efficiency of the optical setup, and SPCM quantum efficiency, respectively. Since $\eta_{\{X \geq 1\}}$ approaches unity as $\bar{N} \rightarrow \infty$, the photon extraction efficiency $\eta_{\text {ext }}$ can be evaluated if both $\eta_{\text {trans }}$ and $\eta_{\text {SPCM }}$ 
are known. $\eta_{\text {trans }}$ is experimentally obtained to be $\sim 0.0208$, which includes transmission of a series of optical filters, lenses, mirrors, and an optical window. From the SPCM datasheet, we assumed $\eta_{\mathrm{SPCM}}=0.245$. Eventually, $\eta_{\text {ext }}$ from the InAs QD located in the silver microcolumnar photon reflectors amounts to $18 \%$. This value is much improved compared with $\sim 1 \%$ for a QD under a planar air/semiconductor interface and $8 \%$ in our previous work. ${ }^{19)}$

Finite-difference time domain (FDTD) simulation in which the QD is located on the center axis of the present 500 nm-diameter pillar shows that $\sim 56 \%$ of photons generated from a quantum dot are extracted to outer airside by the Ag microcolumnar photon reflector. The portion of photon flux integrated over a solid angle defined by the NA of the objective lens was 0.8 (blue area in Fig. 1(f)), which leads to the calculated photon extraction efficiency of $45 \%$. Deviation from the experimentally obtained $18 \%$ is mainly caused by in-plane random positioning of the QD, and also by light scattering due to the residual wavelength-scale roughness at the micropillar sidewalls. It is also shown that, toward the efficient fiber coupling to the present photon source, slightly smaller diameter instead of $500 \mathrm{~nm}$ fabricated in this work will be preferable due to the well-defined single-mode nature of radiation field as shown by the red area in Fig. 1(f). Further reduction in diameter will result in drastic decrease of the photon extraction efficiency due to a "cut-off" at $\sim 350 \mathrm{~nm}$.

At the lower excitation power, reduction of the $g^{(2)}(0)$ value was observed. Furthermore, we have found that $g^{(2)}(0)$ can be further improved by selecting the appropriate excitation energy. In Fig. $4, g^{(2)}(0)$ values measured under two excitation energies are summarized. In this case, we examined a different sample (with almost identical structure) in which the spectral overlap between neighboring dots is almost negligible even at saturated intensity. Under the excitation energy above the emission energy by two LO phonons (quasi-resonant excitation), $g^{(2)}(0)$ showed a drastic decrease of nearly one order of magnitude. Actually, we have observed that the multiphoton probability is suppressed to $1 / 333$ in comparison with the WCP with $g^{(2)}(0)=1$. $^{23)}$ The observed low $g^{(2)}(0)$ value owes possibly to the suppressed absorption in the GaAs barrier layer, which prevents the generation of uncorrelated photons. This is realized by reducing the absorption cross section by means of the below-barrier-bandgap quasi-resonant excitation with additional effect of diminishing GaAs volume in the micro-pillar structures.

In summary, we have introduced a silver microcolumnar photon reflector to enhance the photon extraction efficiency from a quantum dot. A high fabrication yield is realized 
with the aid of a silane coupling agent. We have succeeded in extracting $18 \%$ of the photons emitted from a QD into air and subsequently collected by the first objective lens. A quite low multiphoton probability is also demonstrated. Due to the mechanical robustness of the present planar photon source structure with a Ag microcolumnar photon reflector, highly stable single-photon sources coupled to an optical fiber are promising and now under study.

\section{Acknowledgement}

This work was supported in part by Grants-in-Aid for Scientific Research (S), No. $\sharp 24226007$, and (B), No. $\sharp 24310084$ from the Ministry of Education, Culture, Sports, Science and Technology, SCOPE from the Ministry of Internal Affairs and Communications, Nano-Macro Materials, Devices and System Research Alliance, and the Okawa Foundation for Information and Telecommunications. 


\section{References}

1) M. Sasaki, M. Fujiwara, H. Ishizuka, W. Klaus, K. Wakui, M. Takeoka, S. Miki, T. Yamashita, Z. Wang, A. Tanaka, K. Yoshino, Y. Nambu, S. Takahashi, A. Tajima, A. Tomita, T. Domeki, T. Hasegawa, Y. Sakai, H. Kobayashi, T. Asai, K. Shimizu, T. Tokura, T. Tsurumaru, M. Matsui, T. Honjo, K. Tamaki, H. Takesue, Y. Tokura, J. F. Dynes, A. R. Dixon, A. W. Sharpe, Z. L. Yuan, A. J. Shields, S. Uchikoga, M. Legré, S. Robyr, P. Trinkler, L. Monat, J. -B. Page, G. Ribordy, A. Poppe, A. Allacher, O. Maurhart, T. Länger, M. Peev, and A. Zeilinger: Opt. Express 19 (2011) 10387.

2) N. Namekata, H. Takesue, T. Honjo, Y. Tokura, and S. Inoue: Opt. Express 19 (2011) 10632.

3) E. Waks, C. Santori, and Y. Yamamoto: Phys. Rev. A 66 (2002) 042315.

4) N. Gisin, G. Ribordy, W. Tittel, and H. Zbinden: Rev. Mod. Phys. 74 (2002) 145.

5) K. Takemoto, Y. Sakuma, S. Hirose, T. Usuki, N. Yokoyama, T. Miyazawa, M. Takatsu, and Y. Arakawa: Jpn. J. Appl. Phys. 43 (2004) 933.

6) A. Schwagmann, S. Kalliakos, I. Farrer, J. P. Griffiths, G. A. C. Jones, D. A. Ritchie, and A. J. Shields: Appl. Phys. Lett. 99 (2011) 261108.

7) A. Enderlin, Y. Ota, R. Ohta, N. Kumagai, S. Ishida, S. Iwamoto, and Y. Arakawa: Phys. Rev. B 86 (2012) 075314.

8) S. Strauf, N. G. Stoltz, M. T. Rakher, L. A. Coldren, P. M. Petroff, and D. Bouwmeester: Nat. Photonics 1 (2007) 704.

9) M. Pelton, C. Santori, J. Vučković, B. Zhang, G. S. Solomon, J. Plant, and Y. Yamamoto: Phys. Rev. Lett. 89 (2002) 233602.

10) I. Friedler, C. Sauvan, J. P. Hugonin, P. Lalanne, J. Claudon, and J. -M. Gérard: Opt. Express 17 (2009) 2095.

11) J. Claudon, J. Bleuse, N. S. Malik, M. Bazin, P. Jaffrennou, N. Gregerse, C. Sauvan, P. Lalanne, and J. -M. Gérard: Nat. Photonics 4 (2010) 174.

12) M. E. Reimer, G. Bulgarini, N. Akopian, M. Hocevar, M. B. Bavinck, M. A. Verheijen, E. P. A. M. Bakkers, L. P. Kouwenhoven, and V. Zwiller: Nat. Commun. 3 (2012) 1.

13) M. Munsch, N. S. Malik, N. Gregersen, J. Bleuse, E. Dupuy, A. Delga, J. Mørk, J. -M. Gérard, and J. Claudon: arXiv: 1209.5269v1.

14) D. Rülke, D. M. Schaadt, H. Kalt, and M. Hetterich: Appl. Phys. Lett. 100 
(2012) 251101.

15) O. Gazzano, S. Michaelis de Vasconcellos, K. Gauthron, C. Symonds, P. Voisin, J. Bellessa, A. Lemaître, and P. Senellart: Appl. Phys. Lett. 100 (2012) 232111.

16) H. Sasakura, X. Liu, S. Odashima, H. Kumano, S. Muto, and I. Suemune: arXiv: 1210.3123.

17) M. Davanço, M. T. Rakher, W. Wegscheider, D. Schuh, A. Badolato, and K. Srinivasan: Appl. Phys. Lett. 99 (2011) 121101.

18) H. Kumano, H. Nakajima, S. Ekuni, Y. Idutsu, H. Sasakura, and I. Suemune: Adv. Math. Phys. 2010 (2010) 391607.

19) H. Nakajima, S. Ekuni, H. Kumano, Y. Idutsu, S. Miyamura, D. Kato, S. Ida, H. Sasakura, and I. Suemune: Phys. Status Solidi C 8 (2011) 337.

20) Photon correlation measurement revealed that the electron capture rate into the QD is about 5 times faster than that of electron-hole pair. The origin of this unbalanced capture rate could be a presence of potential barrier for holes in wetting layer as discussed in D. A. Yarotski, R. D. Averitt, N. Negre, S. A. Crooker, A. J. Taylor, G. P. Donati, A. Stintz, L. F. Lester, and K. J. Malloy, J. Opt. Soc. Am. B 19 (2002), 1480. The detailed dynamics of the charge state transfer in the present QD will be discussed elsewhere.

21) R. H. Brown and R. Q. Twiss: Nature 177 (1956) 27.

22) K. Kuroda, T. Kuroda, K. Sakoda, K. Watanabe, N. Koguchi, and G. Kido: Phys. Rev. B 79 (2009) 035330.

23) H. Nakajima, H. Kumano, H. Iijima, and I. Suemune: Appl. Phys. Lett. 101 (2012) 161107. 